\title{
UPDATE OF THE LHC ARC CRYOSTAT SYSTEMS LAYOUT AND INTEGRATION
}

\author{
J-C. Brunet, P. Cruikshank, R. Ostojic, A. Poncet ${ }^{\#}$, P. Proudlock, P. Rohmig, L. Tavian, \\ B. Skoczen, CERN, Geneva, Switzerland
}

\section{Abstract}

Since the LHC Conceptual Design report's publication in October 1995 [1], and subsequent evolutions [2], the LHC Arc Cryostat System has undergone recently a number of significant changes, dictated by the natural evolution of the project.

Most noteworthy are the recent decisions to route the large number of auxiliary circuits feeding the arc corrector magnets in a separate tube placed inside the cryostat with connections to the magnets every half-cell.

Further decisions concern simplification of the baseline vacuum and cryogenic sectorization, the finalization of the design of the arc cryogenic modules and the layout of the arc electrical distribution feedboxes.

The most recent features of the highly intricate cryogenics, magnetic, vacuum and electrical distribution systems of the LHC are presented.

\section{INTRODUCTION}

The Large Hadron Collider (LHC) to be installed in the LEP tunnel at CERN will produce two proton beams of $7 \mathrm{TeV}$ energy for head on collisions in 4 points around the circumference. The machine is subdivided in 8 octants, each one comprising a standard arc layout composed of 54 optical half-cells, flanked on each side by dispersion suppressors and experimental and machine insertions (injection, dump, cleaning, RF, etc.). The standard arc and dispersion suppressors are housed in a common cryostat of diameter 914-mm (36"), together with some adjacent insertion devices, depending on the octant. The cryostat length of an octant varies between $2.7 \mathrm{~km}$ and $3 \mathrm{~km}$, and is bounded at each extremity by electrical current feedboxes.

With the passage from version 5.0 to the current baseline version 6.0 of the optics, additional families of independently powered magnets have been added in the dispersion suppressors and matching sections, leading to a large increase of the number of electrical circuits integrated into the arc cryostat. The cryogenic distribution line (QRL) housing various headers servicing the main stream of cryomagnets runs parallel to the arc cryostat, and is fed at the 4 even points of the machine from cryoplants installed at ground and underground levels. The basic cooling segments fed from cryogenic service modules connected to the QRL installed every cell are: a half-cell (beam screens, magnet support posts) and a cell (magnet string helium filling, cooling at $1.9 \mathrm{~K}$, and discharge).

\section{ARC CRYOSTATS LAYOUTS}

A schematic of the arc cryostat between octants $1 \& 2$ is given in Figure 1. Other arcs differ slightly in length and composition, depending on the octant.

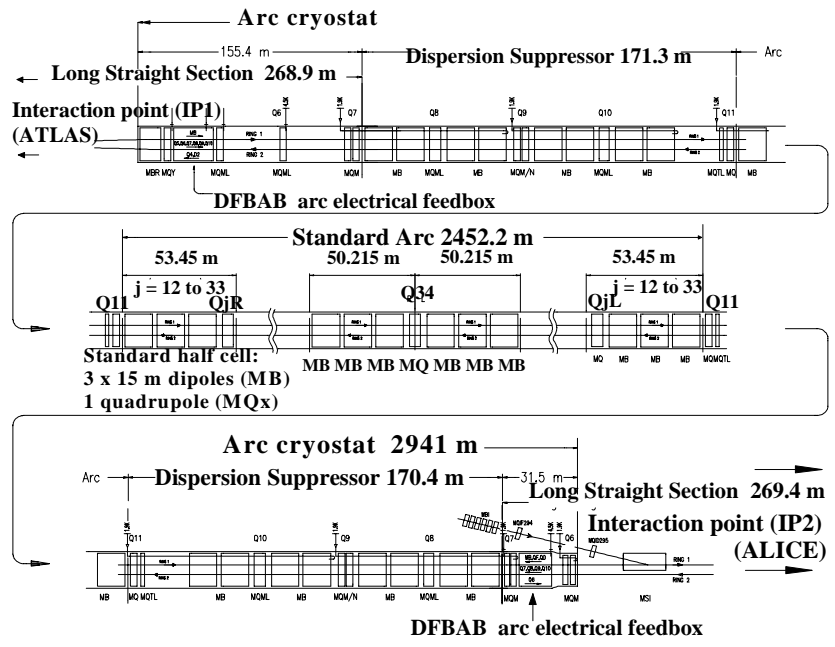

Figure 1: Layout of the Arc Cryostat between Octants 1 \& 2-Optics V6.0.

The basic repetitive magnetic segment of the arc is the half-cell of 53.4-m length providing $45^{\circ}$ phase advance for the beams [5]. It is composed of 3 two-in-one $9 \mathrm{~T}$ (ultimate) dipoles of 15-m length, a two-in-one quadrupole and various sets of correcting magnets. The Short Straight Section (SSS) housing the quadrupole, sextupole, octupole, dipole orbit correctors, and beam position monitors is flanked every cell by a cryogenic service module housing service piping for quench discharge into the cryogenic service line (QRL), phase separation for $1.9 \mathrm{~K}$ heat exchanger cooling, etc. The core of the arc cryostat system is the regular arc, composed of 46 standard half-cells $(2452.2 \mathrm{~m})$, flanked by the dispersion suppressors $(170 \mathrm{~m})$. It is linked to the QRL every cell $(107 \mathrm{~m})$, which provides cooling at pressurized superfluid helium temperature of $1.9 \mathrm{~K}$. The left and right extremities include matching section quadrupoles, beam separation dipoles, and the electrical distribution

${ }^{\#}$ Email: alain.poncet@cern.ch 
feedboxes. Some of these assemblies operate at $4.5 \mathrm{~K}$ in saturated helium, provided by the QRL.

\subsection{Arc magnetic systems}

The main lattice is made up of FODO cells and the two rings are connected in series. Each of the eight arcs of the machine is separately powered. There are 154 twinaperture main dipoles connected in series per arc and when powered to their ultimate current of $13 \mathrm{kA}$ have a stored energy of $1.33 \mathrm{GJ}$. The focussing and defocusing functions are separated and also require $13 \mathrm{kA}$.

The dispersion suppressors at the extremities of the arcs have individually powered quadrupoles of the same type as in the matching sections of the experimental insertions. They require $6 \mathrm{kA}$ and each aperture is individually fed giving up to 12 circuits.

Spool piece correctors are associated with every main dipole, and correct their higher order multipoles errors. At the moment, correction of b3, b4 and b5 is foreseen. The correctors are connected in series for each type and aperture giving a total of 6 circuits. However, spare positions have been left in one of the two types of dipole in the case that additional correctors are required.

The Short Straight Sections (SSS) contain a series of lattice corrector magnets requiring $600 \mathrm{~A}$. There are families of lattice sextupoles, octupoles, trim quadrupoles, skew quadrupoles and skew sextupoles. In all there are typically between 16 and 32 circuits fed from each end of the arcs.

\subsection{Arc electrical distribution}

The circuits associated with the main dipoles (dipole plus spool pieces), as well as the two quadrupole circuits are fed through the cold masses of all magnets. Bus bars are installed in slots in the cold masses. The dipoles use 2 bus bars of $7.4 \mathrm{~mm}^{2} \mathrm{NbTi}$ with $300 \mathrm{~mm}^{2}$ of copper stabilizer. Cold diodes are connected across each dipole magnet and energy is extracted, in case of a quench, at an initial rate of $125 \mathrm{~A} /$ second. The quadrupoles use 4 bus bars of $7.4 \mathrm{~mm}^{2} \mathrm{NbTi}$ with $180 \mathrm{~mm}^{2}$ of copper. Again by-pass diodes are used and the initial discharge rate is $320 \mathrm{~A} /$ second. The dipole corrector magnets use 20 monolithic rectangular conductors with a cross section of $3 \mathrm{~mm}^{2}$ and a copper to superconducting ratio of 9:1.

The individually powered quadrupoles and the array of corrector circuits associated with the SSS need to by-pass the dipoles and a link is provided along the 53 meters of the half-cell. For the $600 \mathrm{~A}$ circuits a flexible multi-wire cable is used containing 36 superconducting wires with a diameter of $1.6 \mathrm{~mm}$ and a ratio $\mathrm{Cu} / \mathrm{SC}$ ratio of $9.5: 1$. The outer diameter of the complete cable is $15.5 \mathrm{~mm}$. A similar cable is used for the $6 \mathrm{kA}$ circuits containing 18 conductors and having an external diameter of $23 \mathrm{~mm}$. These need to be installed in a tube containing helium running from one SSS to another. The number and type of cable varies around the machine according to the local needs.

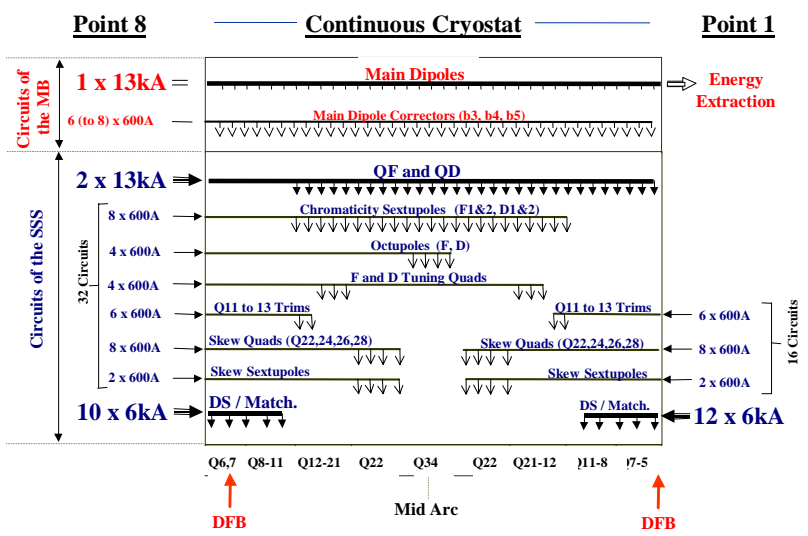

Figure 2: Electrical superconducting circuits feeding the arc cryostat superconducting magnets. The SSS $600 \mathrm{~A}$ circuits are routed in the external auxiliary bus bar tube.

\subsection{Arc cryostat design}

The string of superconducting magnets operates in a static $1.9 \mathrm{~K}$ superfluid helium bath pressurized at $1.3 \mathrm{bar}$ absolute [3]. The heat load budget to $1.9 \mathrm{~K}$ is composed of static heat losses through cryostat components (radiation, conduction through support posts, vacuum barriers, etc.) and dynamic loads resulting from resistive heating in splices between cables, beam losses into the $1.9 \mathrm{~K}$ bath, synchrotron radiation and RF image current power deposited on the beam screen at $5-20 \mathrm{~K}$. To minimize the static heat load to the $1.9 \mathrm{~K}$ level an allwelded aluminum thermal shield operating at $60 \mathrm{~K}$ is installed. The thermal shield is covered by 30 layers of multi-layers insulation (MLI) pre-fabricated blankets to reduce thermal radiation load. The option of an actively cooled shield operating at the sufficient cooling power installed, and of its additional complication.

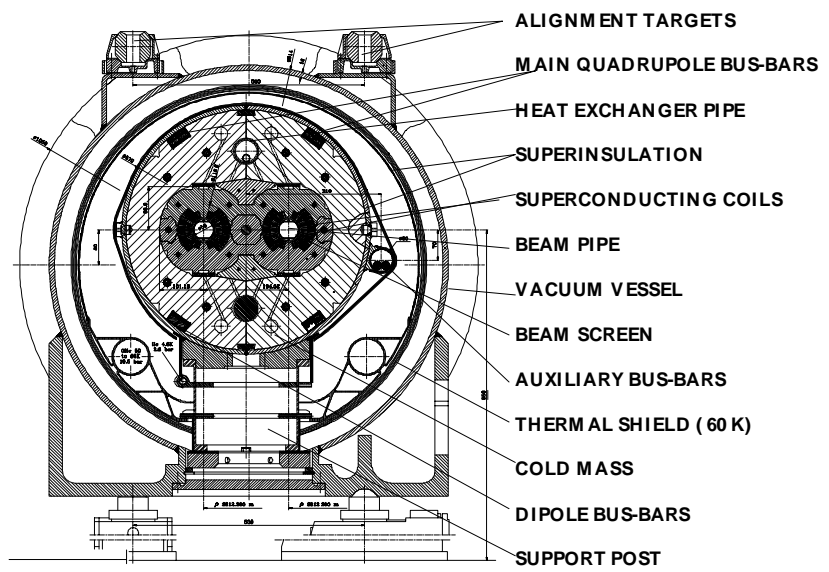

Figure 3: 2D cross-section of the arc cryostat, through one dipole.

The cold mass is simply wrapped with 10 layers of MLI, to reduce conductive heat losses in case of degraded vacuum. 
This MLI covers the auxiliary bus bar tube, thermalized to the cold mass via metallic supports.

The transverse dimensions of magnet cold masses with its shield have been minimized to fit in an insulating vacuum tank of diameter $914 \mathrm{~mm}$ (36" standard pipe dimension), see Figure 3.

The 15-m long cryodipole external supporting system has been improved to obtain higher positioning precision. It is composed of three high-precision jacks distributed over the length of the dipole, at the longitudinal position of the internal supports (cold posts).

Figure 4 shows the integration of the various arc cryostat systems in a zone of interconnection between magnets, cryogenic service module and transverse connection to the QRL.

The routing of superconducting cables feeding the lattice correctors installed in the SSS (octupoles, sextupoles, skew quadrupoles, etc.) is done via a $50 \mathrm{~mm}$ O.D. tube, thermalized to cold masses via metallic supports, connected each half-cell to the corresponding magnetic elements. Thermal contraction during cool down is taken care of by flexible hoses located at each interconnect. The continuous $53.5-\mathrm{m}$ cable unit lengths will be inserted after installation of the cryomagnets in the machine. This solution, compared to previous designs where routing was done inside the cold masses, permits a large reduction of passive electrical interconnections, and yields flexibility, simplifications and reduction in costs.

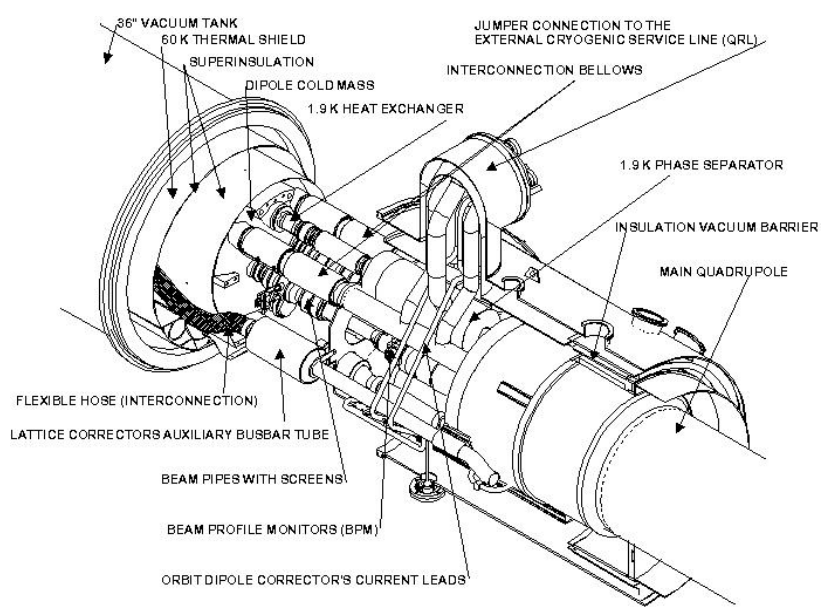

Figure 4: Interconnection of cryogenic, electrical, vacuum and magnetic systems between a lattice quadrupole and a dipole.

\subsection{Sectorization of sub-systems}

To limit commissioning times (e.g. leak search), some intervention times for repair (e.g. defective protection diode) [8], and to limit risks in case of catastrophic vacuum and/or cryogenic failure (e.g. release of cold helium in the tunnel), the $2.7-\mathrm{km}$ arc cryostat cryogenic and insulation vacuum systems are sectorized every 2 cells $(214 \mathrm{~m})[4,6]$. A previous option for full sectorization in 4 subsectors of the arc cryostat included beam vacuum cold valves, permitting replacement of faulty magnets without having to warm-up a full sector. This option has been abandoned in view of its operational complexity [7].

Sectorization is done by means of hydraulic dams-or plugs-installed around bus bars in interconnects, and vacuum barriers in the corresponding SSS.

\section{LHC ARC - CRYOGENIC AND VACUUM BASELINE DESIGN}

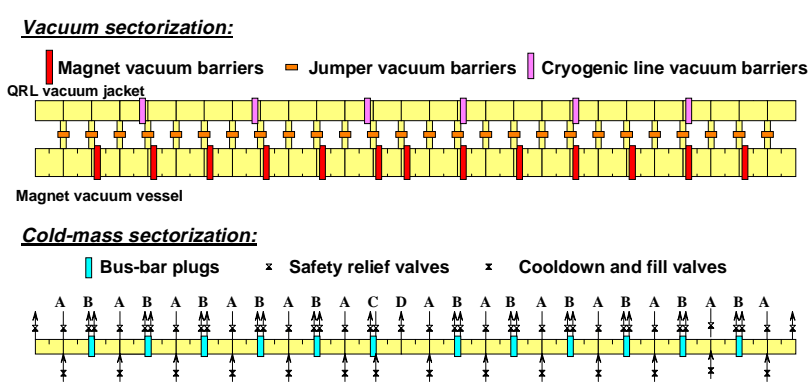

列 Figure 5: Sectorization of the insulation vacuum systems and cold masses in the LHC arc cryostat.

The arc cryostat has a cold mass of more than 5000 tons. One cryoplant at the corresponding even point will be able to cool down and warm-up such a mass via the QRL in about 24 days. For some interventions for repair such as replacement of defective diodes or instrumentation feedthroughs, this sectorization will allow to cool down/warm-up only small stretches of the arc cryostat ( 6 cells) in only about 8 days.

\section{ACKNWLEDGEMENTS}

A large design, development and testing effort is being pursued to integrate all LHC intricate systems in the LHC arc cryostat, and credit goes to the whole of the LHC project staff.

\section{REFERENCES}

[1] The Large Hadron Collider, conceptual design, CERN/AC/95-05 (LHC) (http://www.cern.ch/CERN/LHC/YellowBook95/LHC95/LHC95.html)

[2] L. Tavian et al, A. simplified cryogenic Distribution Scheme for the LHC, ICEC/ICMC'97, Portland, USA, August 97 (LHC Project Report 143 - http:///preprints.cern.ch/cgibin/setlink?base=preprint\&categ=cern\&id=LHCPROJECT-REPORT-143)

[3] Ph. Lebrun, Superfluid Helium Cryogenics for the LHC, ICEC 15, 1994, CERN AT/94-18 (LHC Note 274)

[4] O. Gröbner, The LHC Vacuum System, PAC, Vancouver, 1997 (LHC Project Report 181- http://preprints.cern.ch/cgi-bin/setlink?base=preprint\&categ= cern\&id=LHC-PROJECT-REPORT-181)

[5] J-C. Brunet et al, Mechanical Design and Layout of the LHC Standard Half-Cell, PAC, Vancouver, 1997 (LHC Project Report 111 http://preprints.cern.ch/cgi-bin/setlink?base=preprint\&categ=cern\&id=LHC-PROJECTREPORT-111)

[6] Cryogenic and Vacuum Sectorization of the LHC Arcs, M. Bona et Al, LHC Project Report 60 (http://preprints.cern.ch/cgi-bin/setlink?base= preprint\&categ= cern\&id=LHC-PROJECT-REPORT-60)

[7] A. Poncet, T. Wikberg, Summary Report of the Workshop on LHC Arc Sectorization, LHC Project Note 127, 1998 (http://preprints.cern.ch/cgi-bin/setlink?base=lhcnot\&categ=Note\&id=project-note-127)

[8] M. Chorowski et al, Preliminary risk analysis of the LHC cryogenic system, LHC Project Note 177, 1999

(http://preprints.cern.ch/cgi-bin/setlink?base= lhcnot\&categ=Note\&id=project-note-177) 